\title{
Observação Materna: Primeiros Sinais do Transtorno do Espectro
}

\author{
Autista Maternal \\ Bibiana Massem Homercher* \\ Universidade Franciscana - UFN, Santa Maria, RS, Brasil \\ ORCID: https://orcid.org/0000-0002-3832-7899 \\ Laís Smeha Peres** \\ Universidade Federal de Santa Catarina - UFSC, Florianópolis, SC, Brasil \\ ORCID: https://orcid.org/0000-0001-9193-3831 \\ Liziane Falleiro dos Santos Arruda*** \\ Universidade Franciscana - UFN, Santa Maria, RS, Brasil \\ ORCID: https://orcid.org/0000-0002-1007-034X \\ Luciane Najar Smeha**** \\ Universidade Franciscana - UFN, Santa Maria, RS, Brasil \\ ORCID: https://orcid.org/0000-0002-3068-3776
}

\section{RESUMO}

Os indicadores de risco para o desenvolvimento psíquico da criança podem ser identificados nos primeiros meses de vida do bebê. Essa pesquisa teve como objetivo apresentar e discutir os primeiros sinais, observados por mães de bebês que, posteriormente, na fase da infância, foram diagnosticados com Transtorno do Espectro Autista. O delineamento foi quali-quantitativo. Participaram 27 mães de crianças com Transtorno do Espectro Autista. Os dados foram obtidos por meio de uma entrevista semiestruturada e passaram por uma análise de conteúdo. Posteriormente, os sinais referidos no relato das mães foram listados, reagrupados por áreas e submetidos à estatística descritiva. Constatou-se que os sinais mais observados correspondiam à área da linguagem e do comportamento. Na época da observação, as mães não relacionaram as alterações identificadas com a possibilidade de um diagnóstico de Autismo. Elas também, inicialmente, não foram alertadas por profissionais da saúde em relação aos indicadores já apresentados pelos bebês e, consequentemente, a maioria das participantes recebeu o diagnóstico após os 36 meses da criança. Sugere-se que o os profissionais da saúde participem de capacitações sobre os sinais de risco para o desenvolvimento psíquico infantil, com vistas a uma atuação de qualidade, respaldada pelo crescente aporte teórico sobre o tema.

Palavras-chave: autismo, maternidade, saúde materno-infantil, vínculo mãe-filho. 


\title{
Maternal Observation: Early Signs of Autistic Spectrum Disorder
}

\begin{abstract}
Risk indicators for the child's psychic development can be identified in the first months of the baby's life. This research aimed to present and discuss the first signs observed by mothers of infants who were later diagnosed with Autism Spectrum Disorder during childhood. The design was qualitative and quantitative. 27 mothers of children with Autism Spectrum Disorder participated. The data were obtained through a semi-structured interview and underwent a content analysis. Subsequently, the mentioned signs in the mothers' reports were listed, grouped by areas and submitted to descriptive statistics. It was observed that the most observed signs corresponded to the area of language and behavior. At the time of observation, the mothers did not relate the changes identified with the possibility of a diagnosis of Autism. They were also initially not alerted by health professionals about the indicators already presented by the babies and, consequently, the majority of the participants received the diagnosis after 36 months of the child. It is suggested that health professionals participate in training on risk signs for children's psychic development, with a view to a quality action, supported by a growing theoretical contribution on the theme.
\end{abstract}

Keywords: autism, maternity, maternal and child health, mother-child bond.

\section{Observación Materna: Primeras Señales del Trastorno del Espectro}

\begin{abstract}
Autista
RESUMEN

Los indicadores de riesgo para el desarrollo psíquico del niño se pueden identificar en los primeros meses de vida del bebé. Esta investigación tuvo como objetivo presentar y discutir los primeros signos, observados por madres de bebés que, posteriormente, en la fase de la infancia, fueron diagnosticados con Trastorno del Espectro Autista. El delineamiento fue cuali-cuantitativo. Participaron 27 madres de niños con trastorno del espectro autista. Los datos fueron obtenidos por medio de una entrevista semiestructurada y pasaron por un análisis de contenido. Posteriormente, los signos referidos en el relato de las madres fueron listados, reagrupados por áreas y sometidos a la estadística descriptiva. Se constató que los signos más observados correspondían al área del lenguaje y del comportamiento. En la época de la observación, las madres no relacionaron las alteraciones identificadas con la posibilidad de un diagnóstico de Autismo. También, inicialmente, no fueron alertadas por profesionales de la salud en relación a los indicadores ya presentados por los bebés y, consecuentemente, la mayoría de las participantes recibió el diagnóstico después de los 36 meses del niño. Se sugiere que los profesionales de la salud participen de capacitaciones sobre los signos de riesgo para el desarrollo psíquico infantil, con vistas a una actuación de calidad, respaldada por el creciente aporte teórico sobre el tema.
\end{abstract}

Palabras clave: autismo, maternidade, salud materno-infantil, vínculo madre-hijo. 
Bibiana Massem Homercher, Laís Smeha Peres, Liziane Falleiro dos Santos Arruda, Luciane Najar Smeha

O autismo passou a ser denominado Transtorno do Espectro Autista (TEA) em 2013 e, desde então, pesquisas tem apresentado avanços significativos no conhecimento do espectro, inclusive quanto à possibilidade de identificar sinais de risco nos primeiros meses de vida da criança. Atualmente, no Manual do Diagnóstico e Estatística de Transtornos Mentais - V (DSM - V), lançado em 2013, o Transtorno Global do Desenvolvimento, como era denominado no DSM - IV, passou a ser classificado como TEA. O DSM - V descreve sintomas, tais como déficits persistentes na comunicação e interação social, além de padrões de comportamentos repetitivos (American Psychiatric Association, 2014; Brasil, 2015).

A mudança de nomenclatura do DSM - V amplia a abrangência sobre os sinais de autismo nas áreas da linguagem e comportamento (Forner \& Rotta, 2016). Por ser considerada ampla, a nova classificação permite identificar um número maior de pessoas afetadas. No entanto, isso também gera controvérsias entre os estudiosos do tema, especialmente por não especificar e diferenciar casos anteriormente diagnosticados como Síndrome de Asperger (Cadaveira \& Waisburg, 2015).

A etiologia do TEA, considerado um transtorno do neurodesenvolvimento, ainda não foi elucidada completamente pela comunidade científica, apesar da presença de fortes indícios de bases genéticas. As manifestações do transtorno são diversas e variam de intensidade em cada caso. Além disso, o nível de comprometimento de cada criança é bastante distinto, o que justifica a utilização do termo "Espectro Autista" (Fernandes, Neves, \& Scaraficci, 2006; Pires, 2007; Schmidt, 2017).

No Brasil, os Indicadores de Risco ao Desenvolvimento Infantil (IRDIs) foram validados por meio de um estudo realizado com 700 crianças e coordenado por um grupo de psicanalistas, entre os 250 profissionais envolvidos na pesquisa multicêntrica. Ela teve início no ano de 2000, em parceria com o Ministério da Saúde, e os resultados foram apresentados no ano de 2009. O instrumento é composto por 31 indicadores clínicos de problemas para o desenvolvimento e pode ser aplicado nos primeiros 18 meses de vida do bebê (Kupfer et al., 2009; Kupfer \& Bernardino, 2018).

Outro instrumento que também pode ser utilizado para detectar risco infantil, inclusive por profissionais capacitados de diversas áreas, é o Modified Checklist for Autism in Toddlers (M-CHAT). Trata-se de uma escala de rastreamento de traços de autismo, de baixo custo e fácil aplicação, com questões endereçadas aos pais de bebês entre 18 e 24 meses (Losápio \& Pondé, 2008). Neuropediatras brasileiros também têm utilizado o Childhood Autism Rating Scale (CARS - Escala de Classificação do Autismo na Infância) 
para auxiliar nos diagnósticos de autismo em crianças maiores e na diferenciação entre o TEA e a deficiência intelectual (Pereira, Riesgo, \& Wagner, 2008).

No ano de 2017, entrou em vigor o Projeto de Lei do Senado (PLS) 451/2011 determinando que o Sistema Único de Saúde (SUS) avalie, obrigatoriamente, por meio de protocolo ou outro instrumento, riscos ao desenvolvimento psíquico de crianças com até 18 meses de idade (Lei n. 13.438, 2017). Espera-se que, com a implementação da nova Lei, o país possa atuar de forma preventiva, desde o início das manifestações clínicas no bebê. Entretanto, embora pareça um avanço significativo, a obrigatoriedade de avaliar todas as crianças durante consulta pediátrica pode ser inviável na prática. Segundo Kupfer e Bernardino (2018), as críticas do Ministério da Saúde a essa medida apontam para o risco de processos judiciais contra os profissionais que não detectarem tais sinais. Outra crítica é a falta de articulação com uma política pública de saúde e, além dessas, alerta-se para o risco de patologização, medicalização da infância e possível aumento de resultados equivocados.

Sabe-se que a correta detecção de sinais de riscos pode favorecer o bebê, na medida em que os encaminhamentos para intervenções precoces acontecerão mais cedo, com vistas a um melhor desenvolvimento (Brasil, 2014). Algumas crianças apresentam alteração no desenvolvimento desde os dias iniciais de vida e outras apenas manifestam os primeiros sintomas após os dois anos de vida de suposta normalidade (Pires \& Jou, 2016).

O diagnóstico tardio é uma realidade não somente no cenário brasileiro, mas em todo o mundo (Steyer, Lamoglia \& Bosa, 2018). No entanto, detectar sinais e iniciar precocemente a estimulação é primordial para o melhor desenvolvimento da criança que apresenta risco para o TEA, sobretudo pela possibilidade de atuar no nível das transformações das sinapses neuronais, as quais, nos anos iniciais, ainda estão flexíveis dada a plasticidade neural (Costa, 2014).

Os estudos brasileiros sobre a experiência de maternidade no contexto de um filho(a) com autismo estão aumentando e ajudando a descortinar essa realidade (Christmann, Marques, Rocha, \& Carreiro, 2017; Meimes, Saldanha, \& Bosa, 2015). Todavia, especificamente a respeito da visão materna sobre os sinais iniciais, apesar da importante contribuição de Zanon, Backes e Bosa (2014), ainda pouco se conhece. Nesse sentido, este artigo contribui para elucidar esta importante faceta, na medida em que objetiva apresentar e discutir os primeiros sinais do transtorno observados por mães ao longo do desenvolvimento dos seus bebês que, posteriormente, na infância, foram diagnosticados com TEA. 
Bibiana Massem Homercher, Laís Smeha Peres, Liziane Falleiro dos Santos Arruda, Luciane Najar Smeha

\section{Método}

\section{Participantes}

Trata-se de um estudo quali-quantitativo retrospectivo, de caráter descritivo e exploratório. Participaram, por meio de entrevistas semiestruturadas, 27 mães de crianças com TEA, selecionadas por conveniência. Para preservar a identidade, as participantes foram identificadas pela letra $(\mathrm{M})$, seguida de um número correspondente à ordem de realização das entrevistas. Os nomes dos filhos(as), quando citados nos relatos das mães, foram substituídos pela letra $\mathrm{X}$. Na Tabela 1 constam os dados sociodemográficos das participantes da pesquisa. 
Bibiana Massem Homercher, Laís Smeha Peres, Liziane Falleiro dos Santos Arruda,

Tabela 1

Descrição das participantes entrevistadas

\begin{tabular}{|c|c|c|c|c|c|c|}
\hline Mãe & $\begin{array}{c}\text { Idade da } \\
\text { Mãe }\end{array}$ & $\begin{array}{c}\text { Escolaridade da } \\
\text { Mãe }\end{array}$ & Profissão & $\begin{array}{l}\text { Sexo da } \\
\text { Crianças }\end{array}$ & $\begin{array}{l}\text { Idade atual } \\
\text { da criança }\end{array}$ & $\begin{array}{l}\text { Idade do } \\
\text { diagnóstico } \\
\text { TEA }\end{array}$ \\
\hline M1 & 47 & Ensino Médio & Do Lar & Masculino & 8 anos & 6 anos \\
\hline M2 & 38 & Superior & Médica & Masculino & 5 anos & $\begin{array}{l}1 \text { ano e } 4 \\
\text { meses }\end{array}$ \\
\hline M3 & 38 & Superior & Psicóloga & Masculino & 3 anos & $\begin{array}{l}1 \text { anoe } 7 \\
\text { meses }\end{array}$ \\
\hline M4 & 43 & Superior & Policial Militar & Masculino & 4 anos & 2 anos \\
\hline M5 & 37 & Superior & Policial Civil & Masculino & 9 anos & $\begin{array}{l}2 \text { anose } 4 \\
\text { meses }\end{array}$ \\
\hline M6 & 36 & Ensino Médio & Do Lar & Masculino & 7 anos & 5 anos \\
\hline M7 & 41 & Ensino Médio & Comerciána & Masculino & 11 anos & $\begin{array}{l}3 \text { anose } 4 \\
\text { meses }\end{array}$ \\
\hline M8 & 43 & Superior & Func. Pública & Masculino & 2 anos & 2 anos \\
\hline M9 & 42 & Superior & Func. Pública & Masculino & 12 anos & 3 anos \\
\hline Mlo & 30 & Superior & Do Lar & Masculino & 6 anos & 5 anos \\
\hline Ml & 29 & Superior & Professora & Feminino & 2 anos & 2 anos \\
\hline M12 & 33 & $\begin{array}{l}\text { Superior } \\
\text { Incompleto }\end{array}$ & Professora & Feminino & 10 anos & 15 dias \\
\hline Ml3 & 33 & Ensino Médio & Do Lar & Masculino & 9 anos & 8 anos \\
\hline M14 & 33 & Curso técnico & Do Lar & Masculino & 9 anos & 5 anos \\
\hline M15 & 26 & Superior & Pedagoga & Masculino & 6 anos & 5 anos \\
\hline M16 & 35 & Superior & Empresánia & Masculino & 4 anos & 4 anos \\
\hline M17 & 37 & Supenior & Comerciánia & Masculino & 4 anos & 4 anos \\
\hline M18 & 28 & Superior & Advogada & Masculino & 7 anos & 2 anos \\
\hline M19 & 33 & Superior & Psicóloga & Masculino & 4 anos & 3 anos \\
\hline M20 & 41 & Superior & Professora & Masculino & 10 anos & 2 anos \\
\hline M21 & 40 & Ensino Médio & Do Lar & Feminino & 9 anos & 2 anos \\
\hline M22 & 35 & Superior & Do Lar & Masculino & 7 anos & 3 anos \\
\hline $\mathrm{M} 23$ & 32 & Superior & Autônoma & Feminino & 10 anos & 3 anos \\
\hline M24 & 34 & Superior & Enfermeira & Masculino & 3 anos & 2 anos \\
\hline M25 & 33 & Ensino Médio & Cabelereira & Feminino & 8 anos & 2 anos \\
\hline M26 & 38 & Superior & Administradora & Masculino & 7 anos & 4 anos \\
\hline $\mathrm{M} 27$ & 41 & Superior & Contadora & Masculino & 7 anos & 2 anos \\
\hline
\end{tabular}




\section{Instrumentos}

O material obtido, a partir de uma entrevista semiestruturada, composta por questões abertas sobre o tema, foi transcrito na íntegra. As mães falaram sobre a experiência de ser mãe de uma criança com diagnóstico de autismo. O conteúdo apresentado nesse artigo sintetiza o que foi narrado nas entrevistas pelas mães com base na seguinte questão: "Quais foram suas primeiras observações no sentido de que havia algo diferente no desenvolvimento do seu bebê?" As entrevistas foram realizadas entre os meses de março e dezembro de 2016.

\section{Procedimentos}

\section{Coleta de Dados}

O contato com a primeira mãe ocorreu por intermédio da Associação de Pais na cidade onde foi realizado o estudo, região central do Rio Grande do Sul. As demais participantes foram encontradas mediante a aplicação da técnica Bola de Neve (Vinuto, 2014). A maioria dos filhos diagnosticados com TEA era do sexo masculino: 22 meninos e apenas cinco meninas. Este resultado vai ao encontro do relatado na literatura (Klin, 2006), que descreve maior incidência de autismo em pessoas do sexo masculino, variando de 3,5 a 4,0 meninos para uma menina.

A maioria das mães optou por responder a entrevista na sua residência ou no local onde a criança realizava suas terapias. A coleta de dados teve início após a aprovação do projeto no Comitê de Ética, conforme CAAE n 48427915.6.0000.5306, de agosto de 2015, da Universidade Franciscana.

\section{Análise dos Dados}

As falas transcritas passaram por análise de conteúdo (Bardin, 1977). A categorização do material fundamentou-se no que já é consenso na literatura sobre os sinais de risco para o desenvolvimento psíquico de crianças. Ao final da análise, emergiram cinco categorias: alterações na linguagem, isolamento social, alterações comportamentais, alterações sensoriais e alterações no desenvolvimento motor. Após a análise qualitativa, todos os sinais mencionados pelas participantes em cada uma dessas categorias foram 
listados e submetidos a uma análise estatística descritiva, o que evidenciou dados quantitativos em relação às áreas mais referidas pelas mães.

\section{Resultados e Discussão}

Os sinais mais observados pelas mães participantes foram: alterações da linguagem (35\%); alterações comportamentais, incluindo gritos, hiperatividade e movimentos repetitivos (20\%); isolamento social (18\%); alterações comportamentais e sensoriais (17\%); e perturbações no desenvolvimento motor (10\%). Todos esses indicativos vão ao encontro da descrição do Transtorno do Espectro Autista do DSM-V.

\section{Alterações na Linguagem, no Comportamento e Isolamento social}

As alterações do TEA estão presentes desde os primeiros anos de vida e, geralmente, as crianças apresentam sinais de alerta antes dos três anos. Caracterizam-se por desvios qualitativos na comunicação, na interação social e no uso da imaginação (Mello, 2007). No entanto, a ampla informação sobre o autismo é muito recente no Brasil. Atualmente, na carteira de vacinação do recém-nascido, os pais já encontram informações sobre os sinais de autismo e a importância de detectá-lo precocemente. Paralelamente, as redes sociais, as campanhas de televisão e os movimentos das associações de pais são primordiais para chamar a atenção da população para este problema de saúde, ainda que circulem informações sem respaldo científico e, algumas vezes, equivocadas.

O fato de um bebê evitar olhar para o rosto humano é um sinal importante para o risco de autismo, uma vez que o interesse em buscar o olhar do cuidador já é observado antes dos três meses de idade. Nos casos com TEA, o bebê pode não buscar esse olhar ou buscá-lo com menor frequência (Brasil, 2014). Pesquisa realizada na China investigou oito mil crianças de 18 a 36 meses, por meio de um instrumento que incluía questões sobre sinais precoces do autismo. Os resultados revelaram que aspectos relativos à interação social, tais como monitoramento do olhar, contato visual, interesse pelos pares e idade do primeiro sorriso, tiveram uma presença maior do que outros aspectos para diferenciar crianças com TEA de outras (Huang et al., 2014).

Geralmente, a interação social não é o primeiro sinal que chama a atenção dos pais, embora também tenha aparecido com frequência no relato das participantes deste estudo. A mãe M6, por exemplo, afirmou que "tipo ele não olhava, né? Ele não olhava pra gente". 
Bibiana Massem Homercher, Laís Smeha Peres, Liziane Falleiro dos Santos Arruda, Luciane Najar Smeha

M9 comentou que "chegava na frente dele, olhava nos olhos dele e ele desviava o olhar" e M16 relatou ter sido a avó a primeira a notar a diferença no olhar: "Ela (a avó) observou que ele não olhava muito nos olhos". M27 também fez a mesma observação: "Ele não olhava no olho".

Apesar do autismo se manifestar nos primeiros meses de vida, há relatos de casos em que há um período de desenvolvimento aparentemente típico e, posteriormente, os sintomas começam a se manifestar (Mello, 2007). Algumas mães deste estudo nomearam de "regressão" o que aconteceu com os filhos, embora não exista consenso formal sobre existir ou não alguma regressão no desenvolvimento da criança diagnosticada com TEA. Alguns pesquisadores questionam se há perdas ou estagnação do desenvolvimento (Lampreia, 2013). De acordo com Mello (2007), é frequente pais de crianças com TEA afirmarem que a criança teve um período de normalidade. As mães M9, M10 e M11 relataram que seus bebês apresentavam um desenvolvimento típico nos primeiros anos, mas, com o tempo, surgiram alterações: "Dois anos e meio começou parece a travar, tipo uma engrenagem que para, sabe? Vai parando, vai parando. Aí começou a parar de falar, começou a se fechar, a não ouvir" (M9). "O primeiro aninho a gente não notou muita, muita coisa diferente. Ele olhava, ele ria, ele brincava. Comigo, pelo menos, ele sempre brincou [...] aí depois de um ano assim mais ou menos, ele começou a parar de falar, não falava mais, a gente chamava, ele ignorava. A partir daí ele começou a regredir, regredir, regredir” (M10); “Antes de fazer um ano, ele falava um pouco mais e aí parece que voltou sabe, ele regrediu” (M27).

Apesar da percepção dos pais de prejuízo no desenvolvimento, casos de regressão “pura”, segundo Lampreia (2013), são minoria, em torno de 16\%. De acordo com Backes (2012), as pesquisas que tratam de regressão do desenvolvimento no TEA focam, primordialmente, nos aspectos linguísticos, os quais representam as perdas mais relatadas por mães.

No Brasil, embora o diagnóstico do TEA esteja sendo realizado cada vez mais precocemente, dada a ampla divulgação do tema, é necessário conhecimento para evitar que, os sinais de risco psíquico sejam compreendidos como critério para o estabelecimento de um diagnóstico precipitado de TEA, considerando que nos primeiros anos de vida, a criança ainda está em fase de estruturação da vida psíquica. Por isso, há necessidade de ampliar investimentos na capacitação de profissionais da saúde e educação. Um estudo realizado com enfermeiros e outros profissionais da Estratégia da Saúde da Família, revelou a falta de ações em educação permanente sobre os sinais, sintomas e possíveis intervenções, 
o que implica pouco conhecimento e atraso no acionamento de serviços para o atendimento terapêutico (Nascimento, Castro, Lima, Albuquerque, \& Bezerra, 2018).

Soma-se a isso a constatação de que a maioria dos pais não detecta sintomas comportamentais e cognitivos nos primeiros meses de vida do filho (Porciuncula, 2016). Neste estudo, a mãe M10 comentou que atualmente, ao rever um vídeo em que seu filho brincava na pracinha, não conseguia identificar sinais de autismo naquela idade. Isso mostra que os sinais podem ser imperceptíveis ou, ainda, que algumas mães não os identificaram por falta de conhecimento sobre o assunto.

O TEA, por ser um transtorno complexo, associa sinais característicos do espectro como o transtorno da linguagem, o que inclui ausências de funções pré-linguísticas, dificuldades na coordenação motora e atenção visual. Assim, a primeira infância do autismo, em geral, costuma estar associada a problemas nas habilidades sociocomunicativas, no processamento de contatos faciais e visuais, imitações e comunicação (Forner \& Rotta, 2016).

A aquisição da linguagem depende de um aparato neurobiológico e social que consiga se adaptar às intercorrências, ou seja, é necessário que as estruturas cerebrais tenham um desenvolvimento adaptativo e funcional. A linguagem necessita tanto de aparatos fisiológicos como de estímulos do ambiente (Mousinho et al., 2008). A interação com o universo simbólico surge no ser humano por meio do contato com essa linguagem, e isso o distingue de outros animais. A linguagem produz uma diferenciação entre corpo e sujeito; um animal não pode dizer o que sentiu, vivenciou e percebeu porque o seu corpo não está imerso na linguagem (Levin, 2001).

A aquisição da fala verbal acontece em um contexto comunicativo, no qual a relação e a interação pais/filho são fundamentais. A linguagem oral integra regras complexas de reunião de sons, palavras e frases com algum sentido, que exige um propósito (Freire, 2012). Ao contrário de bebês com desenvolvimento típico, que não demoram para desenvolver atributos que possibilitam a comunicação por meio de sinais não verbais, no TEA, há prejuízos na comunicação, atrasos, alterações na fala e na linguagem (Porciuncula, 2016). Os relatos das mães desse estudo indicaram algumas observações: "Ele não falava muito bem pela idade dele, ele já tinha dois anos e meio e não acompanhava outras crianças na fala e não... não entendia direito" (M1); "Com um ano e meio que ele parou de falar" (M4); "O que nos levou a procurar, a levar ele na neuro, foi a ausência de linguagem” (M6). As mães M7, M11, M14, M16, M20 e M21 também referiram atraso, regressão ou ausência de fala. 
Bibiana Massem Homercher, Laís Smeha Peres, Liziane Falleiro dos Santos Arruda, Luciane Najar Smeha

Destaca-se que não apenas a linguagem verbal é prejudicada no TEA, mas, também, a não verbal, que consiste na troca de sinais gestuais e faciais, postura do corpo e sons não verbais que geram expressões comunicativas (Freire, 2012). Assim, por ser um importantíssimo suporte para a interação, a comunicação, quando prejudicada, afeta a área da socialização, atrapalhando as relações interpessoais, pois há dificuldade tanto de iniciar a interação como de mantê-la (Pereira, Borges, \& Marques, 2015; Schmidt, 2013).

O problema de socialização também está relacionado à dificuldade de demonstrar e compartilhar sentimentos, gostos e emoções (Mello, 2007). Outra característica observada nas crianças com TEA é o modo de brincar, uma vez que preferem brincar sozinhas e podem ser indiferentes à intenção de outro de compartilhar a brincadeira (Martins, 2009). Neste estudo, as mães descreveram o que observaram nesses momentos: "Ele era uma criança isolada, percebia que ele era mais quietinho, percebia que ele não falava" (M5); "As brincadeiras dele eram só dele, que ele gostava muito de brincar de encaixar, ele tinha uma obsessividade pelas letras e tinha dificuldade de interagir, né, com as crianças” (M7); "Não brincava. Praticamente não saía de casa, sempre em cima da cama, era bem isolado" (M13); "Ele não brincava muito com as outras crianças, pois ele se escondia, gostava de brincar sozinho" (M17); "Sempre sentadinho num cantinho assim, brincando com carrinho de roda deitado ou com as mãos ou com qualquer objeto, sem interagir muito” (M18); “Começou a se isolar mais, separação de brinquedo por cor, alinhamento assim de objetos e a falta de socialização" (M23).

Diferentemente de crianças com desenvolvimento típico, aquelas com TEA demonstram menos curiosidade, pouco prazer no contato físico e baixo interesse pela "atenção" de outras pessoas. Nesta fase, elas podem permanecer horas sozinhas, sem chorar ou fazer exigências aos pais, que imaginam ter um filho tranquilo, fácil de cuidar. Os bebês com TEA podem ser calmos, sonolentos ou chorar sem consolo por longos períodos de tempo (Mello, 2007; Porciuncula, 2016). As mães M3, M18 e M20 mencionaram ter observado esses sinais antes dos dois anos de vida do bebê: "Começou a ter dificuldade pra dormir, se debatia assim à noite, dormia pouco, muito agitado também” (M3); "No início, quando bebê, ele era muito calminho, só que à tardinha ele geralmente já começava a chorar” (M18); “Tu observa que a criança chora muito" (M20).

O excesso de choro ou a demasiada quietude são sinais importantes, embora isoladamente não indiquem risco para o desenvolvimento do bebê. Após o diagnóstico e ampliação do conhecimento sobre o TEA, as mães M18 e M20 conseguiram associar esse comportamento inicial como um indicativo de que algo já não estava bem com o bebê nos 
Bibiana Massem Homercher, Laís Smeha Peres, Liziane Falleiro dos Santos Arruda, Luciane Najar Smeha

primeiros 12 meses de vida. Outras pesquisas sobre o tema revelaram que, de forma retrospectiva, a maioria dos pais reconhece algumas dificuldades antes do segundo ano de vida da criança (Zanon, Backes, \& Bosa, 2014).

\section{Alterações Sensoriais e Alterações no Desenvolvimento motor}

Além de déficits na comunicação, na linguagem e na interação social, há outros sinais não recorrentes, como os prejuízos na integração da percepção sensorial. Outras esferas são afetadas no TEA, tais como dificuldades em ficar exposto a estímulos sensoriais devido a uma hipersensibilidade, uma movimentação motora excessiva ou uma calmaria demasiada, distúrbios na coordenação motora e disfunções na alimentação e no sono (Machado, Palladino, Levis, \& Cunha, 2016). Geralmente, as mães acreditam que, por não atender aos chamados, a criança possa ter problemas auditivos, o que está relacionado a percepções alteradas e insensibilidade a certos estímulos: "Não falava, então a gente não dava muita atenção também pra gente [...] será que ele tem algum problema no ouvido ou será que ele... né (M7); "Não responde aos chamados, não obedece aos comandos" (M8); "Começou a se fechar, a não ouvir, eu chamava ele [...]. Primeiro sintoma foi assim, eu chamava e ele nem... como se não tivesse falando com ele" (M9); "Tu chamava ele, ele não te atendia, a gente achou que era surdo" (M27).

As alterações sensoriais também podem ser observadas por insensibilidade a certos odores e sabores, bem como a temperaturas exacerbadas ou à dor (Foster, 2010). De acordo com Schwartzman e Araújo (2011), algumas vezes ocorre falta de resposta ao som, como uma surdez, e em outras há uma reação a pequenos ruídos, o que se denomina inconsistência. São respostas desproporcionais, que podem variar entre ir aos prantos quando alguém toca no seu corpo a não reagir de modo sensível ao sofrer alguma queda. As mães M5 e M17 narraram essas alterações na experiência com seus filhos: "A gente começou a perceber mesmo alguma alteração... com um ano de meio, por aí, que ele começou a ter crises [...] ele explodia, batia a cabeça, ele se dava tapa” (M5); "Dificuldade de se ambientar nos lugares, dificuldade assim, de tu ir no lugar onde ele nunca foi, de ter várias pessoas, de ter muito barulho e ele se esconder" (M17).

Em relação ao desempenho motor, apesar de não ser um critério importante para o diagnóstico de TEA, algumas mães deste estudo referiram alterações neste aspecto: “Hoje em dia, lembrando, eu já sei que o X sentou um pouco atrasado, né... Ele não engatinhou, ele não abanava, não... não batia a palminha, isso no tempo certo"; (M2) "Era um pouco 
mole assim, que ele tinha hipotonia, então a gente já ficou meio assim" (M3). Já M12 e M15 referiram um atraso significativo nas aquisições motoras: "Ela já teve que ter mais atenção pelo fato de caminhar só com 2 anos e meio. Então ela começou, começou primeiro aos 6 meses, que ela já não sentava, que é quando a criança já começa a sentar sozinha, ela não tinha a força" (M12); "Foi uma criança que demorou pra sentar, demorou pra caminhar, demorou pra engatinhar e demorou, sabe” (M15).

Apesar de poucos estudos terem investigado atrasos no desenvolvimento motor das crianças com diagnóstico de TEA, é possível afirmar que elas possuem desvios nos padrões motores em relação a indivíduos típicos (Catelli, D’Antino, \& Blascovi-Assis, 2016). A heterogeneidade sintomatológica é ampla e pode se manifestar nas áreas da comunicação e comportamento de forma independente. Isso significa que determinada área pode apresentar comprometimento significativo, ao mesmo tempo em que outra se desenvolve de modo semelhante ao de uma criança com desenvolvimento típico (Schmidt, 2017).

Tendo em vista as singularidades de cada caso dentro de um amplo espectro, os programas de capacitações de profissionais da saúde pública precisam ser efetivos, tendo como alicerce a identificação das diferenças de natureza qualitativa no desenvolvimento sociocomunicativo e comportamental de crianças com indícios de problemas no desenvolvimento. Para isso, é necessário articular, de forma prática, os conceitos teóricos desenvolvimentais e neurodesenvolvimentais, a fim de utilizá-los como parâmetros no reconhecimento de desvios e/ou atraso que podem variar de sutis a graves (Steyer, Lamoglia, \& Bosa, 2018). A complexidade dos sinais e sintomas demanda aprofundar o conhecimento a esse respeito, sobretudo entre os profissionais que acompanham o crescimento dos bebês nas ações do programa Estratégia Saúde da Família (ESF). Desse modo, a formação continuada da equipe, por meio de programas validados de capacitação, oportunizará a observação e a escuta mais atentas e acolhedoras, auxiliando os pais na detecção dos sinais de risco para o desenvolvimento do bebê.

\section{Considerações Finais}

Essa pesquisa teve por objetivo apresentar e discutir as observações maternas quanto às manifestações precoces dos sinais de alerta para o TEA. Nos resultados, destacase a área da linguagem como a mais referida pelas mães, seguida de alterações de comportamento e isolamento social. Embora a maioria das participantes tenha observado sinais de alerta para o TEA nos primeiros meses de vida dos filhos, elas não identificaram a 
possibilidade de suas observações indicarem problemas no desenvolvimento do bebê, o que pode ser atribuído a diversos fatores: falta de conhecimento sobre o desenvolvimento típico esperado em cada faixa etária, negação de que o(a) filho(a) poderá não se desenvolver da forma como imaginaram e confiança no conhecimento dos profissionais da saúde, especialmente dos pediatras que acompanhavam os bebês.

As mães relataram que o período entre as observações iniciais e o diagnóstico conclusivo foi permeado por incertezas e angústia. Considerando os filhos das 27 participantes, dez foram diagnosticados com quatro anos ou mais, idade considerada tardia para o início da intervenção em estimulação precoce, especialmente porque as mães tinham ensino médio completo ou curso superior, o que revela mais acesso à informação e condições econômicas favoráveis para atendimento em serviços de saúde especializados.

$\mathrm{Na}$ época em que os filhos com TEA eram bebês, as mães tinham pouco ou nenhum conhecimento sobre o autismo. Apesar do nível de escolarização, predominava uma representação adquirida por meio de filmes, nos quais há personagens com autismo com comprometimento severo. Por isso, os primeiros sinais observados pela mãe precisam do amparo do olhar de profissionais da saúde, o que poderá favorecer a identificação, com vistas a antecipar a intervenção de auxílio para desenvolvimento psíquico do bebê. $\mathrm{Na}$ medida em que o SUS, amparado na Lei n. 13.438 (2017), implementar investimentos em capacitações, será possível viabilizar e difundir o conhecimento sobre os sinais de alerta para o TEA, bem como ampliar o uso do protocolo de Indicadores de Risco ao Desenvolvimento Infantil (IRDIs), para o acompanhamento da saúde psíquica nos primeiros 18 meses do bebê.

Para que isso aconteça, são também necessários investimentos por meio de uma gestão pública que acredite e incentive uma prática preventiva no âmbito da saúde mental infantil no Brasil. Assim, a identificação precoce de que algo não vai bem com o bebê poderá ser reconhecida com eficiência já nas suas primeiras manifestações, favorecendo a orientação adequada aos pais e o encaminhamento para os serviços de estimulação da rede pública e/ou privada.

Ressalta-se que o reconhecimento de sinais de risco no desenvolvimento do bebê indica a necessidade de estimulação antecipada, o que não significa estabelecer um diagnóstico de autismo precipitadamente. É necessário intervir com respaldo científico, sem perder a prudência, considerando que uma predição equivocada implicará efeitos iatrogênicos. Esse aspecto precisa ser destacado nos programas de formação profissional no âmbito da Atenção Primária em saúde. 
Bibiana Massem Homercher, Laís Smeha Peres, Liziane Falleiro dos Santos Arruda, Luciane Najar Smeha

Como limitação deste estudo reconhece-se que o delineamento retroativo oportunizou que as mães ativassem suas lembranças com relação ao desenvolvimento inicial dos filhos, mas os fatos recordados podem ter sofrido influência da experiência atual com seus filhos. Além disso, a maioria das participantes, diante do diagnóstico de TEA, buscou conhecimentos teóricos sobre os sinais de risco no bebê, de modo que os fatos por elas relatados nesse estudo podem ter sido recordados sob interferência de informações obtidas posteriormente por meio da literatura e/ou de sentimentos que emergiram ao se lembrarem dos primeiros meses de vida dos filhos. Assim, sugere-se a realização de novos estudos com desenho metodológico semelhante, com vistas a dialogar com os resultados apresentados nesse artigo.

\section{Referências}

American Psychiatry Association. (2014). DSM-5: Diagnostic and statistical manual of mental disorders (5th ed.). Washington, DC: American Psychiatric Association.

Backes, B. (2012). Regressão da linguagem, desenvolvimento sociocomunicativo e perfil sintomatológico de crianças com transtorno do espectro autístico (Dissertação de Mestrado). Universidade Federal do Rio Grande do Sul, Porto Alegre, RS, Brasil. Recuperado

de https://www.lume.ufrgs.br/bitstream/handle/10183/62391/000869415.pdf?sequence $=1$

Bardin, L. (1977). Análise de conteúdo (70a ed). França: Press University de France.

Brasil. (2014). Diretrizes de atenção à reabilitação da pessoa com transtornos do espectro do autismo (TEA). Brasília, DF: Ministério da Saúde. Recuperado de http://bvsms.saude.gov.br/bvs/publicacoes/diretrizes_atencao_reabilitacao_pessoa_a utismo.pdf

Brasil. (2015). Linha de cuidado para a atenção às pessoas com transtornos do espectro do autismo e suas famílias na Rede de Atenção Psicossocial do Sistema Único de Saúde. Brasília, DF: Ministério da Saúde. Recuperado de http://bvsms.saude.gov.br/bvs/publicacoes/linha_cuidado_atencao_pessoas_transtor no.pdf

Brasil (2017). Lei n. 13.438, de 26 de Abril de 2017. Altera a lei n 8.069, de 13 de julho de 1990 (estatuto da criança e do adolescente), para tornar obrigatória a adoção pelo sistema único de saúde (SUS) de protocolo que estabeleça padrões para a avaliação 
Bibiana Massem Homercher, Laís Smeha Peres, Liziane Falleiro dos Santos Arruda, Luciane Najar Smeha

de riscos para o desenvolvimento psíquico das crianças. Brasília, DF: Presidência da República. Recuperado de http://www.planalto.gov.br/ccivil_03/_ato20152018/2017/lei/L13438.htm

Cadaveira, M., \& Waisburg, C. (2015). Autismo, guía para padres y profesionales. Buenos Aires, AR: Paidos Iberica.

Catelli, C. L. R. Q., D’Antino, M. E. F., \& Blascovi-Assis, S. M. (2016). Aspectos motores em indivíduos com transtorno do espectro autista: Revisão de literatura. Cadernos de Pós-Graduação em Distúrbios do Desenvolvimento, 16(1), 56-65. Recuperado de http://pepsic.bvsalud.org/scielo.php?script=sci_arttext\&pid=S151903072016000100007

Christmann, M., Marques, M. A. A., Rocha, M. M., \& Carreiro, L. R. R. (2017). Estresse materno e necessidade de cuidado dos filhos com TEA na perspectiva das mães. Universidade Presbiteriana Mackenzie CCBS. Cadernos de Pós-Graduação em $\begin{array}{lllll}\text { Distúrbios } & \text { do } & \text { Desenvolvimento, } & \text { 8-17(2), }\end{array}$ doi:10.5935/cadernosdisturbios.v17n2p8-17

Costa, D. C. F. (2014). Intervenção precoce no transtorno do espetro do autismo (Dissertação de Mestrado). Escola Superior de Educação João de Deus, Lisboa, Portugal. Recuperado de https://comum.rcaap.pt/bitstream/10400.26/14422/1/DanielaCosta.pdf

Fernandes, A. V., Neves, J. V. A., \& Scaraficci, R. A. (2006). Autismo. Campinas, SP: Instituto de Computação, Universidade Estadual de Campinas. Recuperado de https://www.ic.unicamp.br/ wainer/cursos/906/trabalhos/autismo.pdf

Forner, V. B., \& Rotta, N. T. (2016). Transtorno do espectro autista: Aspectos da intervenção multidisciplinar. In N. T. Rotta, C. A. Bridi Filho, \& F. R. S. Bridi (Orgs.), Neurologia e aprendizagem: Abordagem multidisciplinar (pp. 151-164). Porto Alegre, RS: Artmed.

Foster, O. H. (2010). Autismo em neurologia infantil. In A. Jerusalinsky (Org.), Psicanálise e desenvolvimento infantil (6a ed., pp. 252-261). Porto Alegre, RS: Artes e Ofícios.

Freire, C. M. B. (2012). Comunicação e interação social da criança com perturbação do espectro do autismo (Dissertação de Mestrado). Escola Superior de Educação Almeida Garret, Lisboa, Portugal. Recuperado de http://recil.grupolusofona.pt/bitstream/handle/10437/3050/Tese\%20$\% 20 \mathrm{Cl} \% \mathrm{C} 3 \%$ A1udia\%20Freire.pdf?sequence=1 
Bibiana Massem Homercher, Laís Smeha Peres, Liziane Falleiro dos Santos Arruda, Luciane Najar Smeha

Huang, J. P., Cui, S. S., Han, Yu, Irva, H.-P., Qi, L. H., \& Zhang, X. (2014). Prevalence and early signs of Autism Spectrum Disorder (ASD). Among 18-36 Month Old Childrem in Tianjin of China. Biomedical And Environmental Sciences, 27(6), 453461. doi:10.3967/bes2014.008

Klin, A. (2006). Autismo e síndrome de asperger: Uma visão geral. Revista Brasileira de Psiquiatria, 28(1), 3-11. doi:10.1590/S1516-44462006000500002

Kupfer, M. C. M., Jerusalinsky, A. N., Bernardino, L. M. F., Wanderley, D., Rocha, P. S. B., Molina, S. E., Sales, L. M. S., Estelin. R., Pesaro, M. E., \& Lerner R. (2009). Valor preditivo de indicadores clínicos de risco para o desenvolvimento infantil: Um estudo a partir da teoria psicanalítica. Latin American Journal of Fundamental Psychopathology Online, 6(1), 48-68. doi:10.1590/S1415-47142010000100003

Kupfer, M. C. M., \& Bernardino, L. M. F. (2018). IRDI: Um instrumento que leva a psicanálise à polis. Estilos da Clínica, 23(1), 62-82. doi:10.11606/issn.19811624.v23i1p62-821

Lampreia, C. (2013). A regressão do desenvolvimento no autismo: Pesquisa e questões conceituais. Revista Educação Especial, 26(47), 573-586. doi:10.5902/1984686X10071

Levin, E. (2001). A clínica psicomotora: O corpo na linguagem. Petrópolis, RJ: Vozes.

Losápio, M. F., \& Pondé, M. P. (2008). Tradução para o português da escala M-CHAT para rastreamento precoce de autismo. Revista de Psiquiatria do Rio Grande do Sul, 30(3), 221-229. doi:10.1590/S0101-81082008000400011

Nascimento, Y. C. M. L., Castro, C. S. C., Lima, J. L. R., Albuquerque, M. C. S., \& Bezerra, D. G. (2018). Transtorno do espectro autista: Detecção precoce pelo enfermeiro na Estratégia Saúde da Família. Revista Baiana de Enfermagem, 32, 112. doi:10.18471/rbe.v32.25425

Machado, F. P., Palladino, R. R. R., Lewis, D. R., \& Cunha, M. C. (2016). Surdez e transtornos do espectro do autismo: Reflexões sobre a avaliação fonoaudiológica para o diagnóstico diferencial. Distúrbios da Comunicação, 28(1), 171-174. Recuperado de https://revistas.pucsp.br/index.php/dic/article/view/23157

Martins, A. D. F. (2009). Crianças autistas em situação de brincadeira: Apontamentos para as práticas educativas (Dissertação de Mestrado). Universidade Metodista de Piracicaba, Piracicaba, SP, Brasil. Recuperado de https://www.unimep.br/phpg/bibdig/aluno/visualiza.php?cod=557 
Bibiana Massem Homercher, Laís Smeha Peres, Liziane Falleiro dos Santos Arruda, Luciane Najar Smeha

Meimes, M. A, Saldanha, H. C., \& Bosa. (2015). Adaptação Materna ao Transtorno do Espectro Autismo: Relações entre Crenças, Sentimentos e Fatores Psicossociais. Psico, Porto Alegre, 46(4), 412-422. doi:10.15448/1980-8623.2015.4.18480

Mello, A. M. S. (2007). Autismo: Guia prático (5a ed.). São Paulo: AMA; Brasília: CORDE.

Mousinho, R., Schmid, E., Pereira, J., Lyra, L., Mendes, L., \& Nóbrega, V. (2008). Aquisição e desenvolvimento da linguagem: Dificuldades que podem surgir nesse percurso. Revista Psicopedagogia, 25(78), 297-306. Recuperado de http://pepsic.bvsalud.org/scielo.php?script=sci_arttext\&pid=S010384862008000300012

Pereira, A., Riesgo, R. S., \& Wagner, M. B. (2008). Autismo infantil: Tradução e validação da Childhood Autism Rating Scale para uso no Brasil. Journal of Pediatrics, 84(6), 487-494. doi:10.1590/S0021-75572008000700004

Pereira, C. C. V., Borges, T. A. S., \& Marques, R. R. C. (2015). Tratamento e evolução de crianças autistas atendidas em uma associação de João Pessoa-PB. Revista Ciências Saúde Nova Esperança, 13(1), 77-85. Recuperado de http://www.facene.com.br/wpcontent/uploads/2018/10/v.13-n.1-2015-1.pdf\#page=76

Pires, L. (2007). Do silêncio ao eco: Autismo e clínica psicanalítica. São Paulo, SP: EdUSP; Fapesp.

Pires, S. M. O., \& Jou, G. I. (2016). Identificação precoce do transtorno do espectro autista e diagnóstico diferencial: Estudo de caso. In N. T. Rotta, C. A. Bridi Filho, \& F. R. S. Bridi (Orgs.), Neurologia e aprendizagem: Abordagem multidisciplinar (pp. 5575). Porto Alegre, RS: Artmed.

Porciuncula, R. A. L. (2016). Investigação precoce do transtorno do espectro autista: Sinais que alertam para a intervenção. In N. T. Rotta, C. A. Bridi Filho, \& F. R. S. Bridi (Orgs.), Neurologia e aprendizagem: Abordagem multidisciplinar (pp. 29-54). Porto Alegre, RS: Artmed.

Schmidt, C. (Org.). (2013). Autismo, educação e transdisciplinaridade. Campinas, SP: Papirus.

Schmidt, C. (2017). Transtorno do Espectro Autista: Onde estamos e para onde vamos. Psicologia em Estudo, 22(2), 221-230. doi:10.4025/psicolestud.v22i2.34651

Schwartzman, J., \& Araújo, C.A. (2011). Transtornos do espectro do autismo. São Paulo, SP: Memnon. 
Bibiana Massem Homercher, Laís Smeha Peres, Liziane Falleiro dos Santos Arruda, Luciane Najar Smeha

Steyer, S., Lamoglia, A., \& Bosa, C. A. (2018). A Importância da Avaliação de Programas de Capacitação para Identificação dos Sinais Precoces do Transtorno do Espectro Autista - TEA. Trends in Psychology, 26(3), 1395-1410. doi:10.9788/tp2018.3-10pt

Vinuto, J. (2014). A amostragem em bola de neve na pesquisa qualitativa: Um debate em aberto. Temáticas, 22(44), 203-220. doi:10.20396/temáticas.v22i44.10977

Zanon, R. B., Backes, B., \& Bosa, C. A. (2014). Identificação dos primeiros sintomas do Autismo pelos pais. Psicologia, Teoria e pesquisa, 30(1), 25-33. doi:10.1590/S0102-37722014000100004

\section{Endereço para correspondência}

\section{Bibiana Massem Homercher}

Universidade Franciscana

Rua Silva Jardim, 1175 prédio 16 sala 210, Santa Maria - RS, Brasil. CEP 97010-491

Endereço eletrônico: bibianamh@ hotmail.com

\section{Laís Smeha Peres}

Rua das Moréias, 550, Florianópolis - SC, Brasil. CEP 88053-535

Endereço eletrônico: laissmeha@ hotmail.com

\section{Liziane Falleiro dos Santos Arruda}

Universidade Franciscana

Rua Silva Jardim, 1175 prédio 16 sala 210, Santa Maria - RS, Brasil. CEP 97010-491

Endereço eletrônico: llizijav@ hotmail.com

\section{Luciane Najar Smeha}

Universidade Franciscana

Rua Silva Jardim, 1175 prédio 16 sala 210, Santa Maria - RS, Brasil. CEP 97010-491

Endereço eletrônico: lucianenajar@yahoo.com.br

Recebido em: 01/07/2019

Reformulado em: 16/02/2020

Aceito em: 01/04/2020

\section{Notas}

* Psicóloga. Residente em Saúde Mental - Universidade Franciscana - UFN

** Mestranda em nutrição na Universidade Federal de Santa Catarina - UFSC.

*** Psicóloga. Mestre em Saúde Materno Infantil pela Universidade Franciscana - UFN.

**** Dra. em Psicologia pela PUCRS. Docente no curso de graduação em Psicologia e no Mestrado em Saúde Materno Infantil da Universidade Franciscana - UFN.

Este artigo de revista Estudos e Pesquisas em Psicologia é licenciado sob uma Licença Creative Commons Atribuição-Não Comercial 3.0 Não Adaptada. 